\title{
A relação entre Teoria Crítica da Tecnologia e Slow Design como Contribuição ho Desenvolvimento de Produtos
}

Johelma Pires de Avelar (johpiresdesign@gmail.com) - Programa de Pós-graduação em Design da Universidade do Estado de Minas Gerais (PPGD/UEMG)

Eduardo Romeiro Filho (romeiro@dep.ufmg.br) - Laboratório Integrado de Design e Engenharia do Produto, Departamento de Engenharia de Produção, Universidade Federal de Minas Gerais (LIDEP/DEP/UFMG)

\section{RESUMO}

O modelo de consumo dominante no ocidente parece ter entrado em colapso. Os padrões acelerados de extração de matérias-primas levam à crescente escassez de recursos, além da geração de um impacto ambiental que não é condizente com os limites do planeta. Existem diferentes abordagens na busca de soluções para estes problemas, desde aquelas que privilegiam a inovação tecnológica como forma de manutenção do atual modelo de consumo, mas com redução do impacto ambiental e outras que pressupõem que a tecnologia deve ser guiada pelas necessidades humanas, de forma não predatória e com efetiva mudança no modelo de desenvolvimento atual. Esta perspectiva é difundida pelo movimento slow, que defende que nem sempre o mais rápido pode ser considerado o melhor e que o consumo pelo consumo não tem sentido. Este artigo apresenta uma proposta de abordagem slow para a percepção de necessidades de usuários e definição de características de produtos e serviços a partir da aplicação de ferramentas tradicionais de projeto de produtos ao considerar seis princípios: revelar, expandir, refletir, empenhar, participar e evoluir. É realizada revisão de literatura sobre o tema e apresentado um exemplo de aplicação dos princípios do slow design a um produto de consumo.

Palavras-chave: Slow Design, Sustentabilidade, Metodologia de Projeto, Teoria Crítica da Tecnologia, Movimento Slow. 


\section{INTRODUÇÃO}

Na contemporaneidade, o modelo de consumo e produção segue um padrão onde acontece a autoexploração dos indivíduos, a exclusão do diferente e a falta de percepção de que as relações se tornam cada vez mais virtuais (HAN, 2015). O modelo ocidental de desenvolvimento tem sido alvo crescente de críticas (LIZARRALDE e TYL, 2018) e repensar o estilo de vida dos diversos atores envolvidos nesse contexto social requer do projetista repensar questões culturais, relacionadas ao consumo e a questões políticas e sociais que envolvem o projeto (PINCH e BIJKER, 1984; FEENBERG, 2009; SIMONDON, 2007). O consumo é uma das formas proeminentes de identificação dos indivíduos como sujeitos, quando considerados valores e configurações das subjetividades no ocidente contemporâneo, onde a posse de objetos possibilita parte da constituição da identidade e consumir constantemente se torna uma necessidade. Objetos duráveis passam a ser substituídos por produtos projetados para imediata obsolescência, e a própria subjetividade dos indivíduos se torna efêmera, passa a acontecer em constante construção e no constante perigo de tornar-se inadequada ou ultrapassada (BAUMAN, 2001). Com efeito, o desenvolvimento de novos produtos é considerado um processo fundamental nas empresas (COSTA e WOLFF, 2016).

A concepção de produtos, diante de um contexto de "hiperconsumo", atua em uma lógica de diferenciação social pela posse de bens, que são substituídos em intervalos de tempo cada vez menores (BONDEBJERG e GOLDING, 2004; ARNOLD, 2009; SIRGY, 2001). Contudo, todo esse cenário de fluidez, de velocidade e de relações superficiais, abre espaço para que o designer possa ir além do projeto de interface, possibilita inovar e subverter, passando ao projeto do ritual, das emoções e das relações sociais associadas aos produtos e serviços. Ainda que a forma do produto, associada a outros fatores, seja essencial para seu sucesso (CZIULIK e SANTOS, 2011), cada novo produto é o resultado de um processo influenciado por condições e decisões que envolvem não só questões estéticas, mas também socioeconômicas, tecnológicas, culturais, ecológicas, interesses econômicos e políticos, como também aspirações artístico-experimentais. Ao projeto implica sempre refletir sobre como surgiram tais condições e visualizar seus efeitos sobre os produtos (BURDEK, 2006).

Em nosso trabalho anterior (AVELAR, 2015), foi possível perceber uma discussão embasada na estrutura de duas abordagens teóricas distintas no âmbito dos estudos da Ciência e Tecnologia: (1) a Teoria da Construção Social da Tecnologia (SCOT - Social Construction Of Technology), que considera a partir dos conceitos desenvolvidos por Pinch e Bijker (1984) a 
pertinência de atores sociais relevantes e bifurcações nas trajetórias sociotécnicas e (2) a dos princípios propostos pela Teoria Crítica da Tecnologia, primeiramente postulada por Feenberg (2010), filósofo contemporâneo estadunidense, orientado por Herbert Marcuse, da Escola de Frankfurt. Princípios destas abordagens parecem estar inseridos de forma implícita e não reconhecida formalmente na metodologia projetual, ao longo da história do design e da engenharia. Segundo (SIMONDON, 2007; FENG e FEENBERG, 2008; FEENBERG, 2010), isto permite politizar as relações entre grupos sociais, transformações no mercado e tecnologia, e mostrar de que forma um objeto material pode incorporar não apenas aspectos culturais, representações e ações sociais, mas também relações de poder e ideologia. Isso ocorre não apenas em sua forma, mas no interior de seu próprio código técnico, que define critérios, aparentemente objetivos, de eficiência e adequação. O código técnico, neste caso, é o conjunto de valores e regras socialmente estabelecidas, ideológicas, que automatizam o desenvolvimento da tecnologia estabelecendo uma forma padronizada de enxergar os problemas e as soluções técnicas (FEENBERG, 2009).

A Teoria da Construção Social da Tecnologia (PINCH e BIJKER, 1984) apresenta a ideia de que um objeto técnico, ou um projeto tecnológico, não evoluem de forma linear, ou seja, do menos complexo e imperfeito, para o mais complexo, mais eficiente e mais "perfeito", mas que as trajetórias sociotécnicas são feitas de bifurcações em que os caminhos que se estabilizam são função dos atores sociais envolvidos, dos interesses em jogo, de circunstâncias locais. Assim, um mesmo projeto tecnológico pode levar a produtos técnicos finais muito diferentes. A abordagem SCOT propõe um modelo multidirecional para a análise dos processos que levam à construção da tecnologia: uma abordagem que pretende evitar tanto o determinismo tecnológico, quanto explicações simplistas, rejeitando assim a dualidade entre tecnologia e sociedade, bem como visões externalistas ou essencialistas.

Uma proposta de contraponto à dinâmica econômica e social de hiperconsumo pode ser observada no movimento slow, lançado pelo jornalista italiano Carlo Petrini em 1986 (SINISCALCHI, 2012) e que vem progressivamente ampliando seu escopo (HONORÉ, 2005). Entre estas diversas abordagens, nos interessa particularmente o Slow design (GROSSE-HERING, 2011 e GROSSE-HERING, MASON, et al., 2013), que parte da ótica de que o desenvolvimento de produtos associado aos conceitos do Movimento Slow é capaz de promover uma relação mais adequada das atividades em função do tempo, melhorar e aperfeiçoar o uso dos recursos, valorizar territórios e conhecimentos e promover qualidade de 
vida, dentre outras possibilidades. Estes princípios se tornam um norteador para produtos e serviços desenvolvidos como forma de amenizar a necessidade de aceleração, de promover qualidade de vida dos usuários e melhor uso dos recursos ambientais, sociais e culturais. Propõem uma compreensão da resolução de problemas de projeto sob outras perspectivas e seu impacto social, econômico, político e cultural. (STRAUSS e FUAD-LUKE, 2008).

A abordagem metodológica proposta pelo Slow design apresenta elementos próximos dos princípios da Teoria Crítica da Tecnologia e da Teoria SCOT, agregando também necessidades humanas e sociais para o projeto de design. Os conceitos da TCT e SCOT unidos à abordagem slow, podem permitir a contribuição para uma estrutura projetual, com maior capacidade de agir como um diferenciador social, cultural, político, ambiental, em uma sociedade. Pensar o Slow design como meio para solucionar questões relacionadas ao hiperconsumo pode ser oportuno quando se busca prover qualidade de vida aos indivíduos dentro de um contexto social, ou mesmo, contribuir para que aproveitem melhor o tempo e os espaços (HONORÉ, 2007).

Sob a crença de que poderiam gerar uma mudança de comportamento positiva, de forma a possibilitar qualidade e ações sustentáveis aos processos de produção e consumo dos produtos, (STRAUSS e FUAD-LUKE, 2008) estabeleceram alguns princípios para Slow design, como o princípio (1) Revelar, espaços e experiências na vida quotidiana que muitas vezes não são considerados, incluindo os materiais e processos. O princípio (2) Expandir, que considera as expressões reais e potenciais dos artefatos e ambientes para além das suas funcionalidades percebidas. As características simbólicas e emocionais, além das funcionais devem ser consideradas. O (3) Refletir, artefatos, experiências e ambientes que induzem à contemplação. O (4) Engajar, processos open-source e colaborativos, contam com o compartilhamento, cooperação e transparência das informações para que os projetos possam continuar a evoluir. O (5) Participar, encorajar os usuários a tornarem-se participantes ativos no processo de design, abraçar ideias de convívio e troca, de promoção da responsabilidade social e de melhoramento da comunidade. O (6) Evoluir, reconhecer que experiências mais ricas podem surgir a partir da dinâmica de maturação dos artefatos, ambientes e sistemas ao longo do tempo. Os autores/pesquisadores percebem na relação entre usuários e produtos um sétimo princípio, o Ritual. Ao conseguirem identificar a sugestão de um momento de relaxamento e livre da imposição do tempo, propõem um novo olhar a respeito dos princípios do Slow Design. Dessa forma, os princípios estabelecidos diante da percepção de designers 
ganham uma dimensão de projeto. O Slow Design permite ao designer uma ferramenta capaz de desenvolver produtos que teriam como premissa a garantia à inserção "das esferas do bemestar onde o equilíbrio individual, sociocultural e as necessidades ambientais se interligam" (VORONOVICZ e ZACAR, 2011, p.6).

A proposta para a prática do Slow Design (STRAUSS e FUAD-LUKE, 2008), é aberta à interação e expansão, sendo então, um conceito maleável e passível de desenvolvimento. Com base nessa possibilidade, (GROSSE-HERING, 2013) relata a experiência de desenvolvimento de projeto sob a perspectiva do Slow Design, na tentativa de estabelecer ferramentas características de uso em produtos em escala mais industrial. A pesquisadora pauta que o Slow Design, a partir dos princípios estabelecidos por Strauss e Fuad-Luke (2008), tem condições para gerar nos produtos laço mais duradouro com os usuários, fazendo com que o uso dos produtos e serviços seja mais bem encorajado e mais prolongado, e que tais características podem se estabelecer ao longo de toda a cadeia produtiva, portanto poderão ser caracterizados como produtos e serviços sustentáveis. Busca inicialmente rever um padrão de hiperconsumo, compreendido como regras socialmente estabelecidas, além de repensar a maneira do uso de recursos. Aqui, não só materiais e de processos, mas recursos como o tempo e o prazer de "ser" e "estar", em detrimento de "ter", para com os atores sociais. Isso interfere diretamente na maneira de pensar o projeto de design, nas ações rituais envolvidas, nas relações com as tecnologias e seus impactos, com a maneira como os códigos técnicos (as regras e as relações sociais) são construídos diante de projetos definidos.

Assim, nosso objetivo é apresentar, a partir do exemplo de um produto tecnológico, relações implícita e não reconhecida formalmente no desenvolvimento projetual entre os conceitos propostos pela TCT e SCOT no desenvolvimento de produtos, considerando os princípios da abordagem slow design. Permitindo o projeto do ritual, das emoções e das relações sociais associadas aos produtos e serviços. Após apresentar nesse artigo as relações conceituais entre a TCT, a SCOT e os princípios Slow design, buscaremos exemplificar essas relações a partir da proposição de um caso.

\section{METODOLOGIA}

Buscamos aqui discutir exemplos que apresentem relações entre esses conceitos e mostrem as possibilidades para tal sistematização e contribuições. Para tanto, a metodologia utilizada foi a busca por referencial teórico e a proposição de um caso (YIN, 2001), onde se buscará, segundo Gil, (2008 p.58) “explorar situações da vida real cujos limites não estão claramente 
definidos, e descrever a situação do contexto em que está sendo feita determinada investigação”. Isso, com exemplos de produto tecnológico que pudesse apresentar os elementos propostos no trabalho. Foram consultados os sites do produto, seus aplicativos e resenhas sobre experiência de uso. A escolha do exemplo proposto foi resultado de análise e discussões sobre a apresentação do tema, feita para a turma de mestrado em inovação tecnológica e propriedade intelectual da UFMG, em uma disciplina voltada para sustentabilidade.

\section{RESULTADOS E DISCUSSÃO}

A ANOVA é uma empresa americana que produz fornos de cozimento de precisão, que tem por objetivo declarado "levar a culinária sustentável para todas as cozinhas". Além da versão doméstica do "termocirculador" (que permite cozinhar por um longo período em banho-maria a uma temperatura controlada e sem variações), existe uma versão doméstica de bancada do forno combinado. Tradicionalmente, os fornos combinados só podiam ser encontrados em cozinhas comerciais (restaurantes, hotéis, hospitais etc.), devido aos preços elevados e ao espaço necessário para abrigá-los (a figura 01 mostra as opções de forno pensando as possibilidades e espaços). Em uma cozinha doméstica, para ter possibilidades, seria necessário mais de um forno.

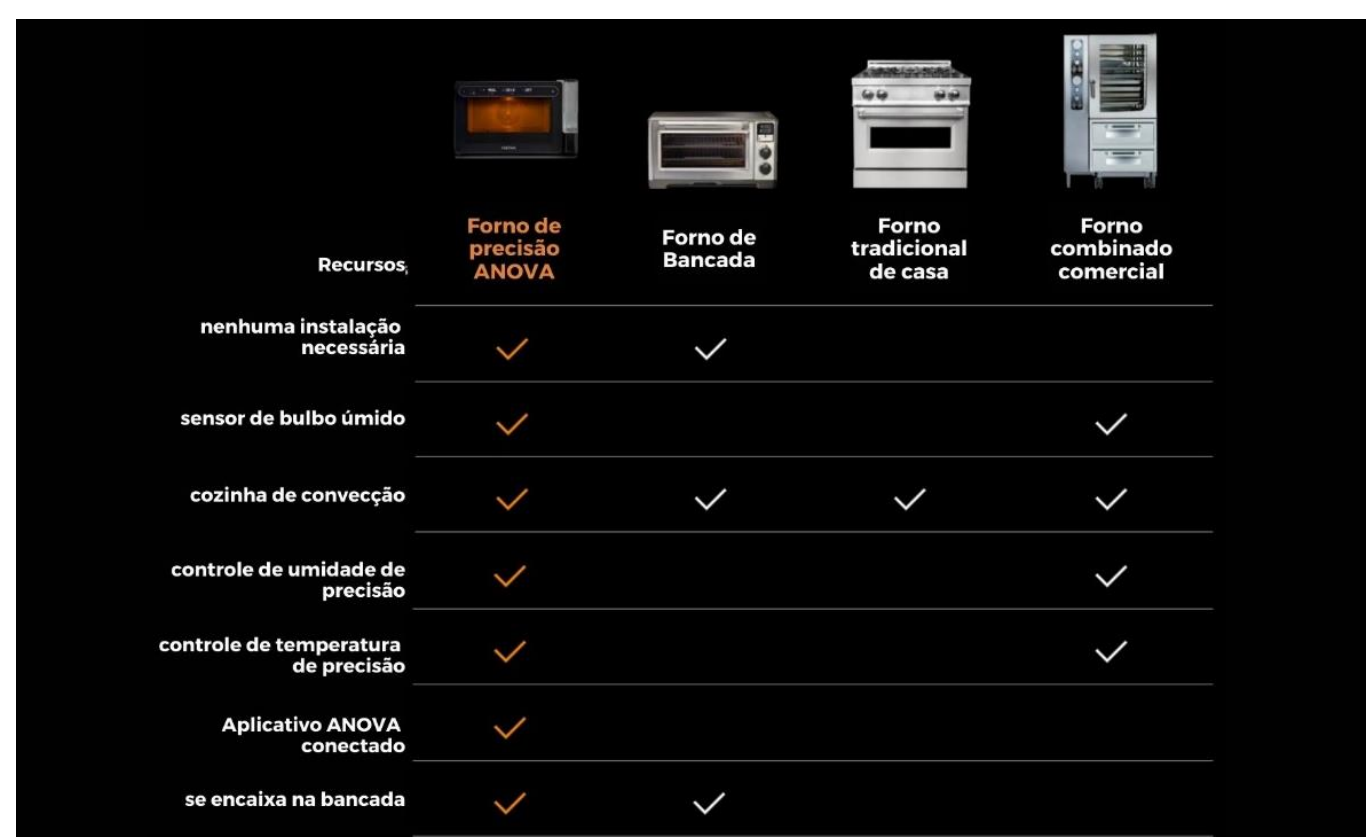

Figura 1 - Comparação entre os tipos de forno existentes, 2021. Fonte: https://anovaculinary.com/ 
Os fornos combinados usam ajustes de temperatura de precisão, emparelhados com a injeção controlada de vapor para criar um ambiente de cozimento ideal, independente do alimento, como podemos ver na figura 2. Agora, segundo a proposta desse produto, é possível aproveitar o poder do controle preciso da temperatura e do vapor em uma cozinha individual, para resultados simples e rituais domésticos, individuais ou que possam ser feitos em menor quantidade (ANOVA, 2021). No aquecimento por convecção é possível assar, grelhar, assar, desidratar ou realizar qualquer outro método de cozimento em calor seco que se esperaria de um forno normal. No controle de umidade e injeção de vapor é permitido aos usuários controlar a umidade relativa dentro do forno por porcentagem ou injetar diretamente vários níveis de vapor durante o cozimento.

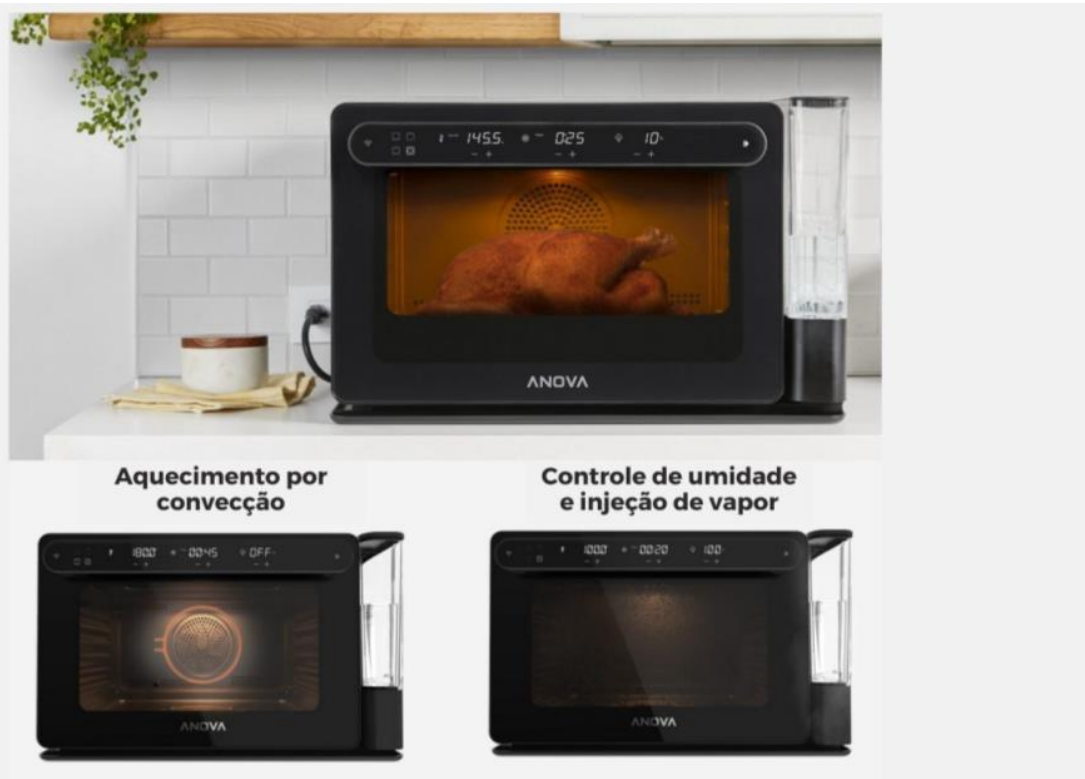

FIGURA 2 - Forno combinado Anova, 2021. Fonte: https://anovaculinary.com/anova-precision-oven/?ab=true

Contudo, a empresa se apresenta mais do que apenas desenvolvedora de dispositivos tecnológicos. Seus fundadores e projetistas se propõem a "transformar a culinária do dia a dia em uma experiência significativa, pessoal e positiva”. Buscam uma maneira de se conectar os produtos e serviços oferecidos com seus usuários e sua comunidade, ao tornar simples cozinhar como um profissional. Assim a proposta é se concentrar no que realmente importa, dentro do ritual. Para tanto, o produto é projetado para pensar em todos os pontos do ritual do cozinhar doméstico e do ambiente de uma cozinha doméstica. Os princípios do slow podem ser percebidos na relação ritual de uso do produto. É importante ressaltar que não podemos afirmar que os produtos ANOVA foram projetados especificamente sob a abordagem Slow 
Design. Contudo, os princípios revelar, expandir, refletir, engajar, participar, evoluir e o ritual são fortemente percebidos.

No princípio revelar, o forno nos revela por meio da facilidade de acesso ao sistema do forno combinado, espaços e experiências no quotidiano do cozinhar que muitas vezes pode ser deixado de lado ou que pode não ser feito por envolver muitas partes incluir muitas etapas, materiais ou processos, o que pode tornar o ritual mais desgastante do que prazeroso. No princípio expandir, o forno vai além da sua função principal (PAZMINO, 2015), apresenta também características simbólicas e emocionais da facilidade, da capacidade de realizar qualquer coisa (mesmo a culinária mais difícil), a possibilidade de reunir sem errar no que se propõe. No princípio refletir o ator social que busca por esse tipo de experiência, por esse tipo de ritual, se estrutura em sua grande maioria em um estilo de vida mais reflexivo, que busca entender melhor o que consome e de onde consome. A empresa nesse sentido, não se propõe a apenas projetar produtos, mas refletir sobre o ritual em questão. Para tanto, podemos encontrar ações da empresa no sentido de fazer o usuário pensar o desperdício de alimentos e propor ações para iniciar mudanças a partir dessas reflexões. No princípio engajar, o produto vai além da tecnologia de cozimento, quando se propõe a conectar o usuário cada vez mais com o seu ritual por meio de aplicativos que ajudam a entender o produto, a controlar o produto, além de descobrir e preparar receitas das mais variadas, como apresentado na figura 3.

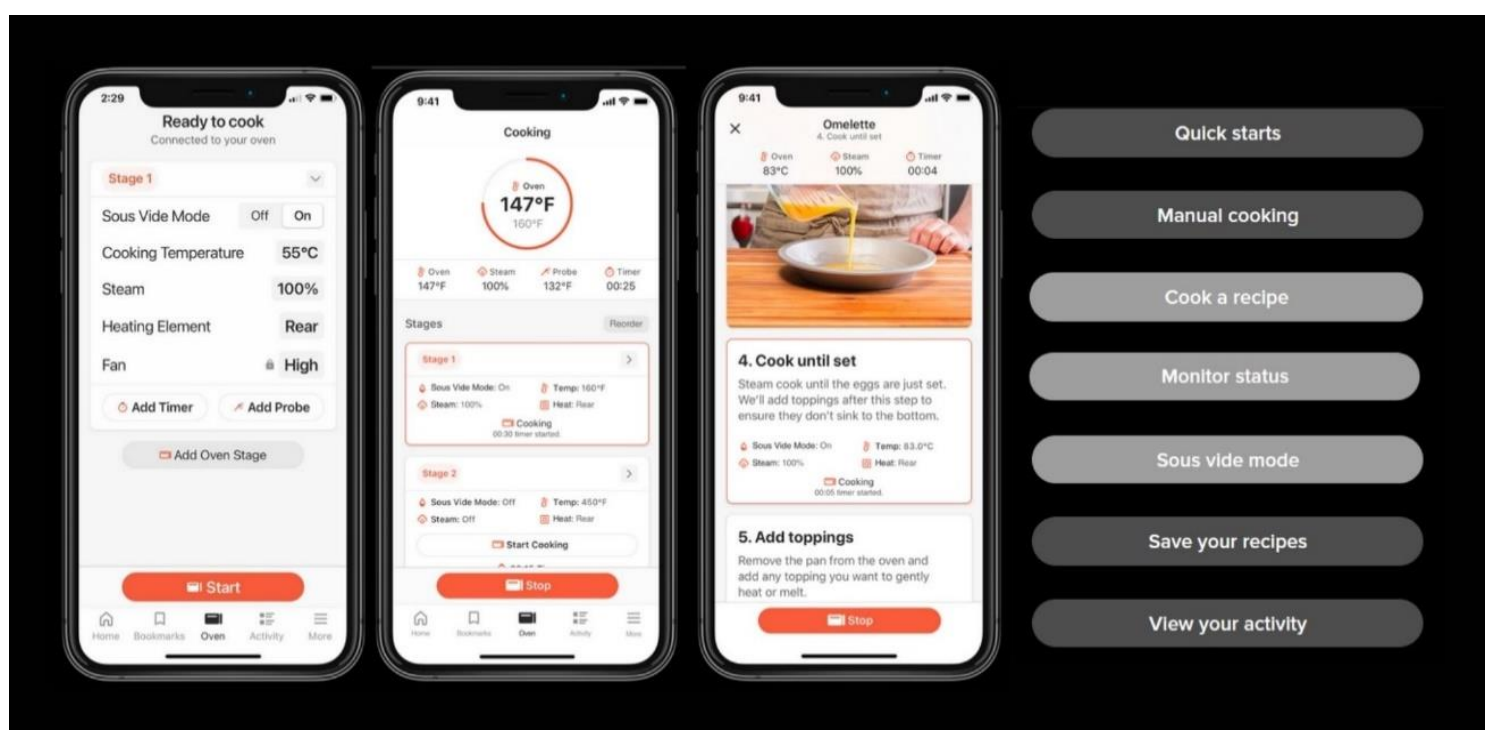

FIGURA 3 - Aplicativo forno combinado Anova, 2021. Fonte: https://anovaculinary.com/anova-precision-oven

No princípio participar, a proposta é de extrapolar a experiência ritual, para uma conexão de troca e de encontro com outras pessoas e experiências, por meio de comunidades que tratam 
do desperdício de alimentos, sobre como comprar sem desperdiçar, descobrir receitas, além um espaço para que se possa compartilhar histórias. No princípio evoluir, é possível perceber que experiências mais ricas podem surgir a partir da dinâmica de maturação desse produto tecnológico. As novas relações com o tempo, novos rituais, o ambiente proporcionado pelo produto, o crescente interesse e reflexão pelo que se consome e como se consome. Futuro interesse em aprimorar conhecimentos, interesses profissionais. São comportamentos rituais que não terminam neles mesmos, mas que se tornam experiências mais profundas e que podem evoluir. Fazendo assim com que o produto também evolua.

O forno combinado, agora adequado para espaços domésticos pode mostrar que a tecnologia, antes permitida apenas para grandes espaços comerciais, agora recebe a influência de projetistas e de atores sociais que pedem pela mudança na forma de preparar e pensar a alimentação, num contexto social onde cada vez mais se busca por entender o que se consome e de onde vem o que se consome, ou seja, a tecnologia influencia e é influenciada pelos atores sociais.

\section{CONCLUSÃO}

Para projetos que buscam por mudanças de comportamento sociocultural, projetos que apoiam um design social, cultural, ambiental e sustentável, é necessário considerar seus atores mais envolvidos, suas necessidades, seus rituais, experiências e histórias. São projetos que devem oferecer a oportunidade para a investigação, o uso mais consciente, o interesse do usuário pelo seu próprio processo, além de promover o uso mais sustentável.

Se o usuário investe tempo para entender, observar e refletir sobre a interação com o produto, o objetivo foi alcançado. O forno ANOVA, mesmo não projetado dentro da estrutura Slow de projeto, apresenta características aqui discutidas de como a ação se torna transparente, o usuário participa do processo, acontece o engajamento, observação, reflexão e interação com o produto. Portanto, pensar o Slow design como meio para solucionar questões relacionadas ao hiperconsumo pode ser vantajoso quando se busca prover qualidade de vida aos indivíduos dentro de um contexto social, ou mesmo, contribuir para que aproveitem melhor o tempo e os espaços (HONORÉ, 2007).

No exemplo do forno combinado ANOVA, é possível ver que o ritual é percebido antes do produto. O forno desenvolvido considera seus atores mais envolvidos, suas necessidades de uso, rituais aos cozinhar, instiga o interesse pelo próprio processo do usuário em cozinhar quando combina os tipos de cozimento com as tecnologias que permitem acompanhar de 
maneira mais precisa a preparação do alimento. Promove um uso mais sustentável, quando elimina a necessidade de outros equipamentos e acessórios e promove o ritual de preparar o alimento, permitindo mais consciência sobre o que se consome.

A ação ritual, ou seja, essa estruturação narrativa de construção de experiência e relação com o tempo das coisas, pelos usuários, então, apresentada como princípio slow design, fícou percebido estar diretamente ligado a conceitos da TCT e da SCOT. Isso se considerarmos como os objetos tecnológicos podem incorporar aspectos culturais, representações e ações sociais, mas também relações de poder e ideologia, e não apenas em sua forma, mas são capazes de participar na estruturação de códigos técnicos, que define critérios, aparentemente objetivos, de eficiência e adequação. E esses critérios, muitas vezes dependem das bifurcações nas trajetórias sociotécnicas

Assim, pensar um projeto sob a abordagem slow design, com as contribuições dos conceitos da TCT e da Teoria SCOT, faz necessário conhecer as relações entre tecnologia e sociedade, além de seu contexto histórico. Tudo isso, relacionado ao momento sociocultural contemporâneo, aos objetos sociotécnicos, à relação de tempo, necessidade e uso, relações de consumo, sustentabilidade, ao atual campo de ação política, aos respectivos atores sociais, ao paradigma da eficiência e códigos técnicos estabelecidos.

Contudo, se faz importante ressaltar que a proposta slow pensa também a relação com a produção dos produtos tecnológicos e não só o processo de criação e desenvolvimento. Pensa o descarte e não só o uso. Mas como já foi dito não podemos afirmar que o produto exemplificado foi desenvolvido sob a abordagem slow. Portanto nossa análise acontece a partir das pesquisas realizadas com o objetivo de discutir a relação entre o slow design e a TCT e SCOT, além de mostrar como elementos sustentáveis de um produto pode ser encontrado no ritual que proporciona.

\section{REFERÊNCIAS}

ARNOLD, C. Ethical marketing and the new consumer. Chichester: John Wiley \&sons LTDA., 2009.

ANOVA APPLIED ELECTRONICS, INC. Anova precision oven. San Francisco, CA, 2021. Disponível em: https://anovaculinary.com/ Acesso em: 08 jun 2021

AVELAR, Johelma Pires de; CASTELFRANCHI, Yurij. Mudanças sociais, objetos técnicos e o design: uma análise sobre os talheres. 2015. 108 f., enc Dissertação (mestrado) -- Universidade Federal de Minas Gerais, Faculdade de Filosofia e Ciências Humanas. Disponível em: < https://repositorio.ufmg.br/bitstream/1843/BUBDA6EJN5/1/mestrado johelma pires junho 2015 vers_o_para_biblioteca_ago_2015.pdf $>$

BAUMAN, Z. Modernidade líquida. Rio de janeiro: Jorge zahar, 2001.

BONDEBJERG, I.; GOLDING, P. European culture and the media. Bristol: Intellect books, 2004. 
BURDEK, B. E. História, teoria e prática do design de produtos. São paulo: Blucher, 2006.

COSTA, S.V., WOLFF, F. Market information management in product design http://dx.doi.org/10.4322/pmd.2016.002 Product, 14(1)47-56, 2016.

CZIULIK, C., SANTOS, F.L. An approach to define formal requirements into product development according to Gestalt principles. http://dx.doi.org/10.4322/pmd.2012.001 Product, 9(2)89-100, 2011.

FENG, P.; FEENBERG, A. Thinking about design: critical theory of technology and the design process. In: VERMAAS, P. E., et al. Philosophy and design: from engineering to architecture. Netherlands: Springer, 2008. p. 105-118.

FEENBERG, A. Critical theory of technology. New york: Oxford university press, 2009.

FEENBERG, A. A teoria crítica de Andrew Feenberg: racionalização democrática, poder e tecnologia. Série cadernos: CCTS - construção crítica da tecnologia e sustentabilidade, Brasília, v. 1, n. 3, 2010.

GIL, A. C. Métodos e técnicas de pesquisa social. 5.ed. São Paulo: Atlas, 2008.

GROSSE-HERING, B. Slow design. University of technology / Institute of positive design. ed. Delft: Philips research eindhoven, 2011.

GROSSE-HERING, B. et al. Slow design for meaningful interactions. CHI 2013: changing perspectives, Paris, 2013. p. 1-11.

HAN, byung-chul. Sociedade do cansaço. tradução de Ênio Paulo Giachini. Petrópolis: vozes, 2015.

HONORÉ, C. In praise of slow: how a worldwide movement is challenging the cult of speed. Londres: Paperback, 2005.

HONORÉ, Carl. Devagar: como um movimento mundial está desafiando o culto da velociade. Rio de Janeiro, Brasil. Editora Record, 2007.

HUGO, VITOR. Forno anova precision oven aposta em funções profissionais para uso caseiro. AGO 30 , 2020. Disponível em: https://pratofundo.com/10053/forno-anova-precision-oven-funcoes-profissionais/ Acesso em: 14 jun 2021

LIZARRALDE, I, TYL, B. A framework for the integration of the conviviality concept in the design process. Journal of Cleaner Production 197(2)1766-1777, 2018.

PAZMINO, A. V. Como se cria: 40 métodos para design de produto. São paulo: Blucher, 2015.

PINCH, T. J.; BIJKER, W. E. The social construction of facts and artifacts: or how the sociology of science and the sociology of technology might benefit each other. Social studies of science, Sage publications, v. 14, n. 3, p. 399-441, aug 1984.

RAY, Joe. Review: anova precision oven. WIRED, dez 2020. Disponível em https://www.wired.com /review/anova-precision-oven/ Acesso em: 11 jun 2021

ROSE, Brent Anova precision cooker review: killer sous vide for everyone. Gizmodo, mar, 2015. Disponível em: https://gizmodo.com/anova-precision-cooker-review-killer-sous-vide-for-eve-1693499013 Acesso em: 11 jun 2021

ROTHMAN, Wilson. From baking to sous vide, this smart steam oven is your new kitchen bff. The wall street journal. Tech | personal technology: review. Oct. 4, 2020. Disponível em https://webreprints.djreprints.com/4922 581368535.html. Acesso em: 11 jun 2021

SIRGY, M. J. Handbook of quality-of-life research: an ethical marketing perspective. Switzerland: Springer, Dordrecht, 2001.

SIMONDON, G. El modo de existencia de los objetos tecnicos. Buenos aires: Prometeo, 2007.

SINISCALCHI, V. Au delà de l'opposition slow-fast. L'économie morale d'un mouvement. Lo squaderno: explorations in space and society, v. 26, n. 7, p. 67-76, dez 2012.

STICKDORN, M.; SCHNEIDER, J. Isto é Design Thinking de Serviços: Fundamentos, Ferramentas, Casos. Porto alegre: Bookman, 2014. 
STRAUSS, C.; FUAD-LUKE, A. The slow design principles: a new interrogative and reflexive tool for design research and practice. In: CIPOLLA, C.; PERUCCIO, P. P. Changing the change design, visions, proposals and tools proceedings. Turin: Allemandi conference press, 2008. p. 01-14.

VORONOVICZ, Priscila; ZACAR, Cláudia. Slow Design e os Requisitos para o Design Sustentável. In: Simpósio nacional de tecnologia e sociedade. UTFPR: Curitiba, 2011.

YIN, Robert K. Estudo de caso: planejamento e métodos. 2 ed. Porto Alegre: Bookman, 2001 